Instability, Chaos and Predictability in Celestial Mechanics and Stellar Dynamics
Copyright 1992 by Nova Science Publishers, Inc. All rights of reproduction in any form reserved. ISBN 1-56072-054-9

\title{
FRAGILITY IN COSMOLOGY AND ASTROPHYSICS
}

\author{
R.K. Tavakol \\ School of Mathematical Sciences, Queen Mary and Westfield College, Mile \\ End Road, London E1 4NS, England
}

ABSTRACT. The theoretical framework adopted in astrophysics and cosmology, in both modelling and the analysis of the observational data, is often implicitly assumed to be that of structural stability. Here, in view of some of the recent results in dynamical systems theory, it is argued that such a framework cannot be assumed a priori and that the fragility framework may instead turn out to be the appropriate framework for the study of certain phenomena in the astrophysical and the cosmological settings. This is motivated by a number of examples from cosmology and a brief discussion of some of the potential domains of its relevance in astrophysics.

\section{INTRODUCTION}

A fundamental assumption usually made in astrophysics and cosmology regarding the nature of the cosmos and hence the theoretical framework within which related hathematical models are constructed and observational data are analysed is that of 'structural stability' (st.st). Briefly, the idea (which was first put forward by Andronov and Pontryagin [1]) amounts to the argument that since 'real systems are stable' and since all mathematical models and the observational data on which they are based are approximations, then 'viable' mathematical models of real phenomena should also be st.st., in the sense of not changing their qualitative behaviours under small ever present errors and perturbations. To make this more precise, recall that by a dynamical system is usually meant a set of coupled nonlinear ordinary differential equations (or their discrete analogue) in the form

$$
\frac{d X^{a}}{d t}=v\left(X^{a}, \mu b\right)
$$


where $X^{a} \in U, U \subset R^{N}, N$ is the dimension of the system and $\mu_{b} \varepsilon R^{M}$ are its 'control parameters'. Now let $V(M)$ be the set of all such vectors fields on a manifold $M$. Then $v \in V(M)$ is said to be structurally stable if there exists a neighbourhood of $v$ in which all vector fields are topological equivalent to $v, i . e$. there exist orientation preserving homeomorphisms that transform the phase space trajectories of one vector field to another. Otherwise we shall call v structurally fragize.

Up to the 1960's, it was generally thought that most dynamical systems (in the sense of genericity, say) would turn out to be structurally stable. This was certainly true for two dimensional systems on compact orientable manifolds [2]. Subsequent developments in dynamical systems theory, however, led to two profound new results, namely that as we go from two to three dimensional deterministic dynamical systems (i) chaotic behaviour becomes possible and (ii) structurally stable systems become rare. The great 'chaos revolution' of the recent years can be said to amount to the ever expanding realisation that the first of these rather abstract outcomes of dynamical systems theory has relevance for a vast range of real phenomena, including extra-terrestrial ones. The aim here is to introduce the idea in [3] that the second of the above results could also turn out to be of significance in the cosmological and the astrophysical domains.

\section{STRUCTURAL FRAGILITY AS A THEORETICAL FRAMEWORK}

A great deal of work in dynamical systems theory eventually led, in the early $1960^{\prime} \mathrm{s}$, to the realisation that structural stability is a rare property among generic dynamical systems of dimension greater than two. Here are two results that highlight this point:

(i) Structurally stable systems are not everywhere dense for systems of type (1) with dimensionl $N \geq 3$ (and for the analogous discrete systems of dimension̄igneater than 2) [4].

(ii) Generic Hamiltonian systems with more than two degrees of freedom are neither integrable nor ergodic, but contain both regions of stochasticity and islands of stability [5].

These results could be interpreted as saying that in dimensions higher than two, 'typical' everyday dissipative systems may turn out to be structurally fragile, in the sense that sma11 changes to such systems could produce enormous effects. For completeness, however, it should be added here that these results do not give any idea as to the measure of fragile systems to be expected. Nevertheless, the fact that almost all mathematical models so far studied are fragile to different degrees supports the idea that fragility framework may be of relevance in variety of settings, including extra- 
terrestrial ones. Furthermore this emphasises the fact that each concrete case must be studied individual1y. In the following sections we briefly discuss some examples which support the potential relevance of structural fragility in the cosmological and astrophysical settings.

\section{FRAGILITY IN ASTROPHYSICS}

The starting point for the study of a great deal of phenomena in astrophysics is the fluid (or the magneto-hydrodynamic) equations. The important characteristing feature of these partial differential equations is their nonlinearity which in turn is mainly responsible for the fact that almost nothing is known about their general solutions. In practice, the common procedure is to forma1ly view such equations as infinite dimensional versions of (1) and then to employ variety of simplifying assumptions to severely reduce them to low dimensional dynamical systems of the type (1). It is worth noting that the numerical routines for solving such equations similarly involve drastic reductions of such equations to finite sets of difference equations. The important point, as far as our discussion here is concerned, is that different sets of simplifying assumptions result in different sets of reductions of the type (1), or its discrete analogue.

Here as an example we briefly discuss the study of ste1lar dynamos in terms of low dimensional deterministic systems. The idea that simple deterministic dynamical systems may be capable of modelling certain features of solar and stellar convective zones has been around for a long time (see for e.g. ref.[6]). Detailed models are diverse, ranging from the three dimensional Lorenz model [7].

$$
\begin{aligned}
& \dot{A}=-A+D B-C B, \\
& \dot{B}=-\sigma B+\sigma A, \\
& \dot{C}=-\nu C+A B,
\end{aligned}
$$

where $A$ and $B$ are the azimuthal components of the vector potential and the mean field, $C$ is the helicity excess due to magnetic helicity, $\sigma$ determines the ratio of characteristic diffusion times of $B$ and $A$, and $v$ is a measure of decay rate of the magnetic helicity due to magnetic diffusion (which is $\ll 1$ in the convective zone) - to higher dimensional reductions such as the (complex) seven dimensional system

$$
\begin{aligned}
& \dot{\mathrm{A}}=2 \mathrm{D}\left(1+k|\mathrm{~B}|^{2}\right)^{-1} \mathrm{~B}-\mathrm{A}, \\
& \dot{\mathrm{B}}=\mathrm{i}\left(1+\omega_{0}\right) \mathrm{A}-\frac{1}{2} \mathrm{iA}{ }^{*} \omega-\left(1+\lambda|\mathrm{B}|^{2}\right) \mathrm{B},
\end{aligned}
$$




$$
\begin{aligned}
\dot{\omega}_{0} & =\frac{1}{2} i\left(A^{*} B-A B^{*}\right)-\nu_{0} \omega_{0}, \\
\dot{\omega} & =-i A B-\omega v .
\end{aligned}
$$

See [8] for the derivation of this system and the relevant notation.

Leaving aside the dynamical details of these systems, the main point for us is that different reductions have widely different modes of behaviour both in their pre and post chaos phases $[7,8]$. As an instructive example we may point out that even a 'trivial looking' asymmetric perturbation of the system (2) which modifies the first of the equations to $A=-A+D B-C B+\varepsilon$, can have drastic consequences for the system such as destroying chaos, even for the constant $\varepsilon$ taking values as small as $0.00075[9]$. Furthermore, the fact that a large proportion of such reductions allow chaotic behaviour does not mean that the attractors involved, and hence their statistical behaviour, are the same. After ali different chaotic systems can have widely different attractors. Even for a fixed simple system such as (2) there is a vast range of possible modes of behaviour as the system's control parameters are varied [10]. In this sense then the 'reduction procedure' and the low dimensional modelling of dynamos may be said to be fragile.

\section{FRAGILITY IN COSMOLOGY}

Recall that the starting assumption in all standard cosmological modelling $(*)$ is Einstien's field equations

$$
R_{a b}-\frac{1}{2} R g_{a b}+\Lambda g_{a b}=\kappa T a b
$$

These equations, despite their seeming simplicity, are a higly nonlinear set of ten partial differential equations about whose general properties very 1 ittle is known. Again to make the problem more manageable, the common practice is to employ observations together with a number of simplifying assumptions (which are not always directly linked to the observations, such as precise symmetry conditions) to reduce the equations (4) to a (low dimensional) dynamical system of the form (1), with parameters such as those characterising inhomogeneity and the cosmological constant acting as the system control parameters $\mu_{b}$. Again in view of the fact that observations can never be made precise and simplifying assumptions are generally present, the question arises as to the nature of the cosmological models that correspond to such approximate models and data. Here, we briefly discuss some examples which support the relevance of fragility framework in this context.

(*) Throughout this article GR is assumed to be the correct theory of gravity within which cosmological medels are sought. 


\subsection{Fragility with respect to the changes in symmetry}

Recall that the assumptions of homogeneity and isotropy reduce Einstein's equations to a dynamical system with $\mathrm{N}=2$, which is only capable of possessing fixed points and periodic solutions. On the other hand relaxing the assumption of isotropy (i.e. allowing unisotropic perturbations) in vacuum wil1 result in dynamical systems with $N>2$ (Bianchi type IX, say) which are capable of chaotic solutions $[11,12]$ (see also [13] for a recent clarification of the nature of such chaos). More generally Fischer et.al [14] have shown that the space of all empty solutions of the Einstien's field equation $E_{e}$, does not possess the structure of a smooth manifold near space-times with Killing vectors, rather it has a conical structure in the sense that locally in the neighbourhood of such solutions, $E_{e}$ has the product structure of a manifold times a cone.

\subsection{Fragility with respect to increase in the number of spatial dimensions.}

\section{Here are some examples:}

(i) persistence of chaos with respect to changes in the number of dimensions

The spatial dimension turns out to be important in determining whether unisotropic Bianchi type IX models mentioned in the previous section are chaotic or not. For example, chaos goes away for spatial dimension $D>3$ if the space time manifold has the product structure of the Kaluza-Klein type with the internal and the external manifolds uncoupled [ 15 ]. On the other hand chaos becomes possible for $3 \leq D \leq 9$ where the manifold is not a product type if off-diagonal terms are included in the metric tensor [16].

(ii) The robustness of exponential solutions in higher dimensions [17, 18]

Consider a $(D+1)$ dimensional Riemannian metrilc

$$
g_{\alpha \beta}=\left(\begin{array}{rrr}
-1 & 0 \\
0 & R_{D}^{2} * g_{i j}
\end{array}\right)
$$

where $\alpha, \beta=0, \ldots, D$ and $i, j=1, \ldots, D$ and ${ }^{*} g_{i j}$ is the metric of a $D$-dimensional space of constant curvature and $R_{D}=R_{D}(t)$ is the D-dimensional scale factor. Employing the (D+1) dimensional Einstein's field equations together with the energymomentum tensor with bulk viscosity in the form

$$
T_{o O}=\varepsilon, T_{i j}=\left(p-\frac{D \zeta(R) \dot{R}_{D}}{R_{D}}\right) g_{i j}
$$


where $\varepsilon$ and $\mathrm{p}$ are respectively the eqnergy density and the isotropic pressure and eliminating $R_{0}$ between the components of the Einstein's equations results in the higher-dimens ional Friedmann equation in the form

$$
\mathrm{D}(\mathrm{D}-1) \frac{\left(\mathrm{K}_{\mathrm{D}}+\dot{\mathrm{R}}_{\mathrm{D}}^{2}\right)}{2 \mathrm{R}_{\mathrm{D}}^{2}}=\varepsilon+\Lambda
$$

where $K_{D}$ is the curvature parameter of the maximally symmetric subspace and $\Lambda$ is the cosmological constant. Now when $\Lambda=K_{D}=0$ and $\zeta(R)=C_{1} R^{2}$, it can easily be checked $[17]$ that equation (7) and the other Finstein's equations possess an exponential solution of the type

$$
R_{D}=R_{0} \exp \left(\frac{t}{C_{2}}\right)
$$

where $C_{1}$ and $C_{2}$ are constants. It is also easy to see that such solutions also survive under non-zero perturbations of $\Lambda$. This then shows that exponential solutions are stable to changes in $D$. This, however, does not ensure the persitence of such solutions in presence of other physically motivated perturbations. As an example we may consider the robustness of such solutions in presence of shear [18]. In that case the generalised Friedmann equation becomes

$$
D(D-1) \frac{\left(K_{D}+R_{D}^{2}\right)}{2 R_{D}^{2}}=\varepsilon+\Lambda+\frac{C \Sigma^{2}}{R_{D}^{2}}
$$

where $C$ is a constant depending upon $D$ and $\Sigma$ is the shear coefficient. It is easy to see that the presence of the shear term prevents equations (9) from having an exponential solution. This is an example of how stability with respect to certain perturbations breaks down as soon as other perturbations are taken into account. For more details and also other examples see $[3,18]$.

\section{CONCLUSION}

Starting from certain recent developments in dynamical systems theory and by considering a number of concrete examples, we have attempted to demonstrate that the relevant theoretical framework for certain astrophysical and cosmological settings may turn out to be that of structural fragility. This could have important consequences for

(i) the construction of unique models to fit the observational data (including error margins) and to satisfy simplifying assumptions (not wholly justified).

(ii) the interpretation of observational data, specially in cosmology where there is no possibility of repetition. 
(iii) the study of real systems for which transience is a permanent feature.

(iv) the understanding of seemingly unrelated modes of behaviour in the astrophysical domain (say dynamos) which may be understood within the single framework of structural fragility.

\section{REFERENCES}

[1] Andronov, A.A. and Pontryagin, L.S. (1937) Dokl. Akad. Nauk. SSSR, 14,247.

[2] Peixoto, M.M. (1962) Topology, 1,101 .

[3] Tavakol, R.K. and E11 is, G.F.R. (1988) Phys.Letts.1.30A, 217 .

[4] Samie, S. (1967) Bu11. AMS, 73,747.

[5] Markus, L. and Meyer, K.R. (1974) Memoires AMS, Number 144 .

[6] Tavakol, R.K. (1978) Nature, 276,802.

[7] Ruzmaikin, A.A. (1985) Solar Phys., 100,125.

[8] Weiss, N.O., Cattaneo, F. and Jones, C.A. (1984) Geophys. Astrophys. Fluid Dynamics, 30,305 .

[9] Cox, S.M. (1990) Phys. Letts. 144A, 325.

[10] Sparrow, C.(1982) The Lorenz Equation. Springer, New York.

[11] Lifshitz, E.M., Lifshitz, I.M., and Khalatnikov,I.M., (1970) JETP, 59,322 .

[12] Barrow, J.D. (1982) Phys. Rep., 85,1.

[13] Burd, A.B., Buric, N. and Tavakol, R.K. (1990) Class. Quantum. Grav. In Press.

[14] Fischer, A.E., Marsden, J.E., and Moncrief, V. (1980). in Essays in General Relativity, F.J. Tipler, ed. (Academic Press, New York).

[ 15] Barrow, J.D. and Stein-Schabes (1985) Phys.Rev.,D32,1595.

[16] Demaret, J., Hanquin, J.-L., Henneaux, M., Spindel,P. and Taormina, A. (1986) Phys.Letts. $175 \mathrm{~B}, 129$.

[17] Wolf, C.(1988) Phys. Letts. 127A, 129.

[18] Farina-Busto, L. and Tavako1, R.K. (1990);: 'An Example of Structural Fragility in Cosmology', Europhysical Letts., 11, 493-497. 